\title{
Collapse of the Electron Gas to Two Dimensions in Density Functional Theory
}

\author{
Lucian A. Constantin, ${ }^{1}$ John P. Perdew, ${ }^{1}$ and J. M. Pitarke ${ }^{2,3}$ \\ ${ }^{1}$ Department of Physics and Quantum Theory Group, Tulane University, New Orleans, Louisiana 70118, USA \\ ${ }^{2}$ CIC nanoGUNE Consolider, Mikeletegi Pasealekua 56, E-20009 Donostia, Basque Country, Spain \\ ${ }^{3}$ Materia Kondentsatuaren Fisika Saila, UPV/EHU, and Centro Física Materiales CSIC-UPV/EHU, \\ 644 Posta kutxatila, E-48080 Bilbo, Basque Country, Spain
}

(Received 26 March 2008; published 3 July 2008)

\begin{abstract}
Local and semilocal density functional approximations for the exchange-correlation energy fail badly in the zero-thickness limit of a quasi-two-dimensional electron gas, where the density variation is rapid almost everywhere. Here we show that a fully nonlocal fifth-rung functional, the inhomogeneous SingwiTosi-Land-Sjölander (STLS) approach, which employs both occupied and unoccupied Kohn-Sham orbitals, recovers the true two-dimensional STLS limit and appears to be remarkably accurate for any thickness of the slab (and thus for the dimensional crossover). We also show that this good behavior is only partly due to the use of the full exact exchange energy.
\end{abstract}

DOI: 10.1103/PhysRevLett.101.016406

PACS numbers: 71.10.Ca, 71.15.Mb, 71.45.Gm

One of the main goals of electronic-structure theory is prediction of the energy of inhomogeneous interacting many-electron systems. Time-independent and timedependent density functional theories (DFT and TDDFT) [1] provide calculable predictions, respectively, for ground and excited states, and are intimately linked together. In these theories, the noninteracting kinetic energy is treated as an exact functional of the occupied Kohn-Sham (KS) orbitals [2], and only the exchange-correlation (xc) energy $E_{\mathrm{xc}}$ and/or potential $v_{\mathrm{xc}}(\mathbf{r})$ have to be approximated. These theories entail a hierarchy of approximations for exchange and correlation. The more sophisticated approximations satisfy many exact conditions, but must still be carefully tested for accuracy and reliability. In this Letter, we test the inhomogeneous Singwi-Tosi-Land-Sjölander (STLS) method [3] for the quasi-two-dimensional (2D) electron gas, a problem that becomes increasingly challenging for density functionals as the true $2 \mathrm{D}$ or zero-thickness limit is approached [4,5].

The ladder classification [6] of ground-state density functionals for $E_{\mathrm{xc}}$ has three complete nonempirical rungs: the local-spin-density approximation (LSDA) [7], the generalized-gradient approximation (GGA) [8], and the meta-GGA [9]. The meta-GGA, which satisfies many exact constraints but still uses to some extent the error cancellation between exchange and correlation, has as ingredients the spin densities $n_{\uparrow}$ and $n_{\downarrow}$, their gradients $\nabla n_{\uparrow}$ and $\nabla n_{\downarrow}$, and the KS noninteracting kinetic energy densities $\tau_{\uparrow}$ and $\tau_{\downarrow}$. These local and semilocal density functionals (LSDA, GGA, and meta-GGA) work for atoms, molecules, solids, and surfaces [10]. They also work for atomic monolayers [11] and other quasi-2D systems [12], but they fail as the true 2D limit is approached [13,14], because of the high inhomogeneity of the electron density along the confined direction. The failure of local and semilocal density functionals to describe the dimensional crossover of the exact $\mathrm{xc}$ functional has been avoided by using nonlocal models such as the weighted-density approximation [15].

The next rung of the ladder is the hyper-GGA (HGGA) [16], a nonlocal-functional approximation which uses the Tao-Perdew-Staroverov-Scuseria (TPSS) meta-GGA [9] ingredients and the conventional exact exchange energies per particle $\epsilon_{x \uparrow}$ and $\epsilon_{x \downarrow}$ [17]. For the xc energy per particle at position $\mathbf{r}$, one writes

$$
\epsilon_{\mathrm{xc}}^{\mathrm{HGGA}}=\epsilon_{x}+[1-a]\left[\epsilon_{x}^{\mathrm{TPSS}}-\tilde{\epsilon}_{x}\right]+\epsilon_{c}^{\mathrm{TPSS}} .
$$

Here $\tilde{\boldsymbol{\epsilon}}_{x}(\mathbf{r})$ is the exact exchange energy per particle in the TPSS gauge [17], and $a(\mathbf{r})$ is a nonlocal functional bounded between 0 and 1 . The second term on the righthand side of Eq. (1) is the static correlation and was built such that, together with the TPSS (dynamic) correlation, it is compatible with exact exchange. The mixing parameter $a(\mathbf{r})$ goes to 1 when exact exchange is much bigger than correlation (for one-electron systems and for the highdensity limit), when the density is rapidly varying, and when an open system has a high fluctuation of electron number in spin-polarized regions at the Hartree-Fock level. HGGA satisfies more exact constraints than any semilocal functional. To balance the full nonlocality of exact exchange, universal empirical parameters are invoked in HGGA correlation and fitted to chemical data. The set of parameters adopted here is for use with TPSS orbitals. We show that this HGGA partially avoids the bad behavior of the semilocal xc density functionals for the quasi-2D electron gas.

The central equation of TDDFT linear response (at frequency $\omega$ ), in which all the objects are functionals of the ground-state density, is a Dyson-like equation for the density-response function $\chi\left(\mathbf{r}, \mathbf{r}^{\prime} ; \omega\right)$ (in atomic units where $e^{2}=\hbar=m_{e}=1$ ) [18]: 


$$
\begin{aligned}
\chi\left(\mathbf{r}, \mathbf{r}^{\prime} ; \omega\right)= & \chi_{0}\left(\mathbf{r}, \mathbf{r}^{\prime} ; \omega\right)+\int d \mathbf{r}_{1} d \mathbf{r}_{2} \chi_{0}\left(\mathbf{r}, \mathbf{r}_{1} ; \omega\right) \\
& \times\left\{\frac{1}{\left|\mathbf{r}_{1}-\mathbf{r}_{2}\right|}+f_{\mathrm{xc}}[n]\left(\mathbf{r}_{1}, \mathbf{r}_{2} ; \omega\right)\right\} \chi\left(\mathbf{r}_{2}, \mathbf{r}^{\prime} ; \omega\right),
\end{aligned}
$$

where $\chi_{0}\left(\mathbf{r}, \mathbf{r}^{\prime} ; \omega\right)$ is the density-response function of noninteracting $\mathrm{KS}$ electrons and is exactly expressible in terms of KS orbitals [19], and $f_{\mathrm{xc}}[n]\left(\mathbf{r}, \mathbf{r}^{\prime} ; \omega\right)$ is the dynamic xc kernel which must be approximated. When $f_{\mathrm{xc}}[n]\left(\mathbf{r}, \mathbf{r}^{\prime} ; \omega\right)$ is taken to be zero, Eq. (2) reduces to the screening equation of the time-dependent Hartree or random phase approximation (RPA). The xc energy can then be calculated using the adiabatic-connection fluctuationdissipation formula [20], which allows TDDFT to produce sophisticated approximations to the ground-state xc energy. In particular, the RPA has been evaluated for the quasi-2D electron gas $[15,21]$ and it reaches the RPA 2D electron gas limit; however, the RPA xc energy per particle $\epsilon_{\mathrm{xc}}^{\mathrm{RPA}}$ for the 2D uniform electron gas underestimates the exact $\epsilon_{\mathrm{xc}}$ by more than 0.02 hartree $/ e^{-}$[15]. In order to go beyond the RPA, various approximations have been constructed for the xc kernel, but they have mainly been taken from the 3D uniform electron gas [23] and do not take the dimensional crossover into account.

The inhomogeneous STLS (ISTLS) [3] is, like the RPA it corrects, a fifth-rung density functional that employs both occupied and unoccupied Kohn-Sham orbitals. The main idea of ISTLS (and STLS [24]) relies on the truncation of the Bogoliubov-Born-Green-Kirkwood-Yvon (BBGKY) hierarchy of the kinetic equations [25], by assuming that the two-particle dynamic pair-distribution function $f^{(2)}\left(\mathbf{r}, \mathbf{p}, \mathbf{r}^{\prime}, \mathbf{p}^{\prime} ; t\right)$ can be expressed in terms of the single-particle distribution function $f(\mathbf{r}, \mathbf{p} ; t)$ as

$$
f^{(2)}\left(\mathbf{r}, \mathbf{p}, \mathbf{r}^{\prime}, \mathbf{p}^{\prime} ; t\right)=g\left(\mathbf{r}, \mathbf{r}^{\prime}\right) f(\mathbf{r}, \mathbf{p} ; t), f\left(\mathbf{r}^{\prime}, \mathbf{p}^{\prime} ; t\right),
$$

where $g\left(\mathbf{r}, \mathbf{r}^{\prime}\right)$ is the static (and momentum-independent) equilibrium pair-correlation function.

Using the linearity and time invariance of the truncated BBGKY equation, Dobson et al. [3] found the following Dyson-like "screening" integral equation for the densityresponse function:

$$
\chi\left(\mathbf{r}, \mathbf{r}^{\prime} ; \omega\right)=\chi_{0}\left(\mathbf{r}, \mathbf{r}^{\prime} ; \omega\right)+\int d \mathbf{r}^{\prime \prime} Q\left(\mathbf{r}, \mathbf{r}^{\prime \prime} ; \omega\right) \chi\left(\mathbf{r}^{\prime \prime}, \mathbf{r}^{\prime} ; \omega\right),
$$

where

$$
Q\left(\mathbf{r}, \mathbf{r}^{\prime} ; \omega\right)=-\int d \mathbf{r}^{\prime \prime} \boldsymbol{\nu}_{0}\left(\mathbf{r}, \mathbf{r}^{\prime \prime} ; \omega\right) \cdot g\left(\mathbf{r}^{\prime \prime}, \mathbf{r}^{\prime}\right) \nabla_{\mathbf{r}^{\prime \prime}} \frac{1}{\left|\mathbf{r}^{\prime \prime}-\mathbf{r}^{\prime}\right|}
$$

Here, $\boldsymbol{\nu}_{0}\left(\mathbf{r}, \mathbf{r}^{\prime} ; \omega\right)$ is a vector response function, which satisfies the equation

$$
\chi_{0}\left(\mathbf{r}, \mathbf{r}^{\prime} ; \omega\right)=\nabla_{\mathbf{r}^{\prime}} \cdot \nu_{0}\left(\mathbf{r}, \mathbf{r}^{\prime} ; \omega\right) .
$$

The equilibrium pair-correlation function $g\left(\mathbf{r}, \mathbf{r}^{\prime}\right)$ is ob- and tained from the fluctuation-dissipation theorem [20]:

$$
g\left(\mathbf{r}, \mathbf{r}^{\prime}\right)=1-\frac{1}{\pi n(\mathbf{r}) n\left(\mathbf{r}^{\prime}\right)} \int_{0}^{\infty} d u \chi\left(\mathbf{r}, \mathbf{r}^{\prime} ; i u\right)-\frac{\delta\left(\mathbf{r}-\mathbf{r}^{\prime}\right)}{n\left(\mathbf{r}^{\prime}\right)} .
$$

Equations (4)-(7) are solved self-consistently, until a converged solution is obtained. This ISTLS scheme yields the exact exchange energy, as does the TDDFT scheme described above [see Eq. (2)], and only the correlation energy is approximated. ISTLS correlation is exact for all oneelectron densities.

For the 2D and 3D uniform electron gases, the STLS approach made a remarkably accurate prediction of the correlation energy over a wide range of densities (for 3D, $1 \leq r_{s} \leq 20$, and for $2 \mathrm{D}, 0.5 \leq r_{s}^{2 \mathrm{D}} \leq 16$ ), as confirmed by quantum Monte Carlo (QMC) calculations (see Table I of Ref. [26] and references therein) [27]. The STLS approximation is also known to yield reasonable ground-state energies for the 1D and 2D Hubbard models in the half filled antiferromagnetic states [28]. Furthermore, the ISTLS, which is a high-level correlated approach that predicts (and does not use as input) the correlation energy of the 3D and 2D uniform gases, has been shown to yield accurate jellium xc surface energies that are close to their LSDA and RPA counterparts, and has been used to demonstrate that a local-density approximation for the particlehole interaction is adequate to describe the surface energy of simple metals [26].

A 2D uniform electron gas is described by the 2D electron-density parameter $r_{s}^{2 \mathrm{D}}=1 / \sqrt{\pi n^{2} \overline{\mathrm{D}}}=\sqrt{2} / k_{F}^{2 \mathrm{D}}$, where $n^{2 \mathrm{D}}$ is the density of electrons per unit area, and $k_{F}^{2 D}$ represents the magnitude of the corresponding 2D Fermi wave vector. The $2 \mathrm{D}$ exchange energy per particle is

$$
\epsilon_{x}^{2 \mathrm{D}}=-[4 \sqrt{2} /(3 \pi)] / r_{s}^{2 \mathrm{D}} .
$$

A realistic interpolation (which uses QMC data) between the high- and low-density limits of the 2D correlation energy per particle is $[13,29]$

$$
\epsilon_{c}^{2 \mathrm{D}}=0.5058\left[\frac{1.3311}{\left(r_{s}^{2 \mathrm{D}}\right)^{2}}\left(\sqrt{1+1.5026 r_{s}^{2 \mathrm{D}}}-1\right)-\frac{1}{r_{s}^{2 \mathrm{D}}}\right] .
$$

For the description of a quasi-2D electron gas, we consider a quantum well of thickness $L$ in the $z$ direction. In the infinite barrier model (IBM) [30] for a quantum well, the KS effective one-electron potential is replaced by zero inside infinitely high potential walls at $z=0$ and $z=L$. Hence, the normalized KS one-electron wave functions and energies at $0 \leq z \leq L$ are

$$
\phi_{l, \mathbf{k}_{\|}}\left(\mathbf{r}_{\|}, z\right)=\sqrt{\frac{2}{A L}} \sin \left(\frac{l \pi}{L} z\right) e^{i \mathbf{k}_{\|} \cdot \mathbf{r}_{\|}}
$$




$$
E_{l, k_{\|}}=\frac{1}{2}\left[\left(\frac{l \pi}{L}\right)^{2}+k_{\|}^{2}\right],
$$

where $A$ represents the normalization area in the $x y$ plane, $l$ is the subband index, and $\mathbf{r}_{\|}$and $\mathbf{k}_{\|}$represent the position and the wave vector in the $x y$ plane. In this model the electrons cannot leak out of the well, so the true 2D electron-gas limit is recovered by simply shrinking the well. When only the lowest level is occupied $\left(E_{1, k_{F}^{2 D}}<\right.$ $E_{2,0}$, i.e., $L<\sqrt{3 / 2} \pi r_{s}^{2 \mathrm{D}}=L_{\max }$ [13]), the density of states of this quantum well begins to resemble the density of states of a $2 \mathrm{D}$ electron gas, the motion in the $z$ direction is frozen out, and the system can be considered quasi-twodimensional.

We would like to contract or expand the electron density $n(z)$ without changing the total number of electrons per unit area. Hence, we perform a one-dimensional scaling of the form $n_{\lambda}(z)=\lambda n(\lambda z)$ [31], which as $\lambda \rightarrow \infty$ yields the true 2D limit. This scaled electron density coincides with the electron density that one would find from Eq. (10) by simply replacing the quantum-well thickness $L$ by $L / \lambda$ [13]. The corresponding exchange and correlation energies per particle, $\varepsilon_{x}\left[n_{\lambda}\right]$ and $\varepsilon_{c}\left[n_{\lambda}\right]$, should satisfy the following scaling relations [13]:

$$
\lim _{\lambda \rightarrow \infty} \varepsilon_{x}\left[n_{\lambda}\right]>-\infty ; \quad 0>\lim _{\lambda \rightarrow \infty} \varepsilon_{c}\left[n_{\lambda}\right]>-\infty .
$$

These equations, which start from those of Ref. [31], are not satisfied by the LSDA, GGA, and meta-GGA.

Here we investigate the performance of the ISTLS approach to describe the IBM quasi-2D electron gas for thicknesses $L$ such that $L<L_{\max }$. We choose quasi-2D electron gases of fixed 2D electron-density parameters: $r_{s}^{2 \mathrm{D}}=2 / \sqrt{3}$ (as in Fig. 1 of Ref. [15]) and $r_{s}^{2 \mathrm{D}}=4$ (as in Figs. 2 and 3 of Ref. [13]). The self-consistent ISTLS scheme [Eqs. (4)-(7)] was solved numerically using occupied and unoccupied orbitals of the form of Eq. (10). Our numerical scheme is similar to the one described in Ref. [26]; however, in contrast to the jellium surfaceenergy calculation reported in Ref. [26], where the ISTLS self-consistent scheme converges rapidly, in the case of the quasi-2D electron-gas calculation the convergence is slow (especially when $L \ll L_{\max }$ ) [32] and special care must be taken to perform the frequency integration of Eq. (7).

Figures 1 and 2 show the results we have obtained, as a function of the quantum-well thickness $L_{\max } / \lambda$, for the exact exchange energy per particle $\varepsilon_{x}$ and the xc energy per particle $\varepsilon_{\mathrm{xc}}$ in the following approximations: LSDA, PBEGGA [8], HGGA, RPA, and ISTLS. We observe that, while local and semilocal density functional approximations (LSDA and PBE-GGA) fail badly in the zero-thickness (2D) limit, both the RPA and the ISTLS nicely recover their 2D counterparts, which in the case of the ISTLS $\left(-0.62\right.$ hartree for $r_{s}^{2 \mathrm{D}}=2 / \sqrt{3}$ and -0.20 hartree for $r_{s}^{2 \mathrm{D}}=4$ [33]) are very close to the prediction of Eqs. (8) and (9) or, equivalently, QMC calculations $(-0.63$ and

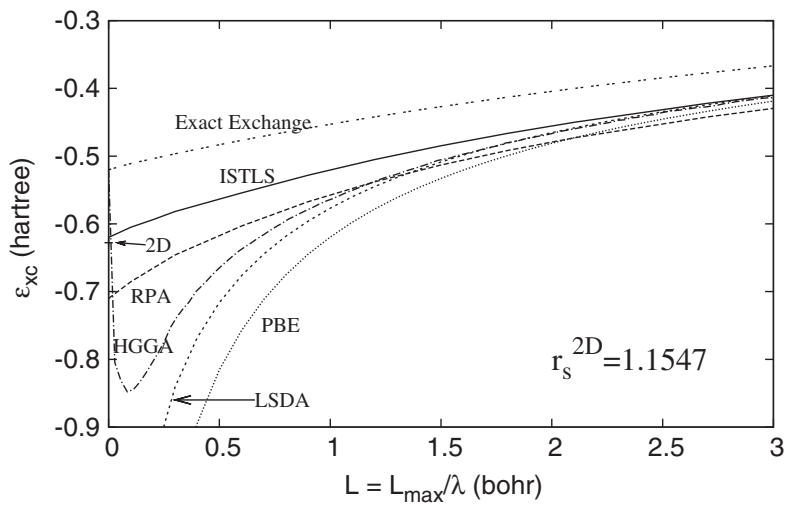

FIG. 1. Exchange-correlation energy per particle of an IBM quasi-2D electron gas of fixed $2 \mathrm{D}$ electron density $\left(r_{s}^{2 \mathrm{D}}=\right.$ $2 / \sqrt{3}$ ), as a function of the quantum-well thickness $L_{\max } / \lambda$ $\left(L_{\max }=4.44\right)$. The 2D limit is from Eqs. (8) and (9). Various density functional approximations have been used (LSDA, PBEGGA, and HGGA), as well as the fifth-rung RPA and ISTLS. The exact exchange energy per particle $\varepsilon_{x}$ is also plotted, for comparison. While the local and semilocal density functionals diverge in the 2D limit, RPA and ISTLS calculations nicely recover the corresponding xc energy of a $2 \mathrm{D}$ electron gas. The HGGA recovers the 2D exact exchange limit; i.e., as in the case of the LSDA and the PBE-GGA, the HGGA correlation energy per particle goes to zero in the $2 \mathrm{D}$ limit.

-0.21 , respectively). The RPA xc energy per particle, however, considerably underestimates $\varepsilon_{\mathrm{xc}}$ for all slab thicknesses [34]. Figures 1 and 2 show that the ISTLS xc energy not only approaches closely the exact $2 \mathrm{D}$ limit but has also the correct behavior in the limit $L \rightarrow L_{\max }$, which is expected to be well described within the LSDA, GGA, and HGGA. The meta-GGA curves, not shown in Figs. 1 and 2, are found to be very close to their GGA counterparts. We note that the HGGA greatly improves over GGA (and meta-GGA) and is more accurate than RPA for almost all values of the slab thickness.

Most of the functionals tested in Figs. 1 and 2 have been (exact exchange, LSDA, PBE) or could be (HGGA, ISTLS) useful in condensed-matter physics and quantum chemistry. For all of those except LSDA and ISTLS, the

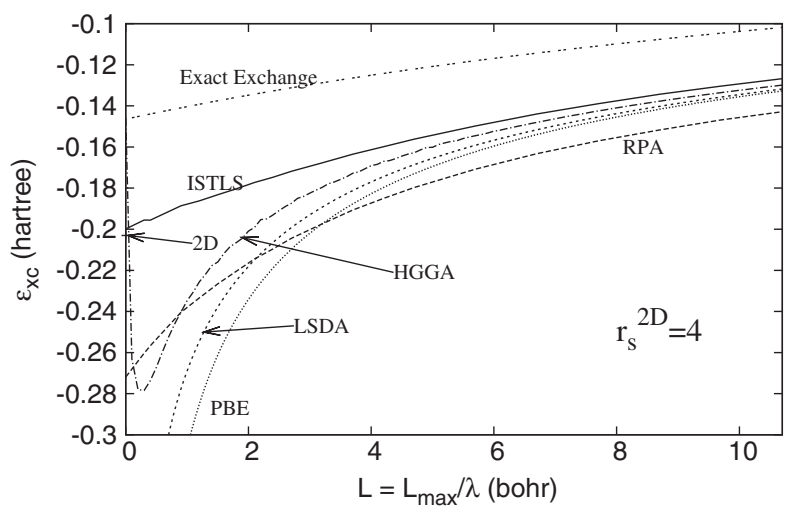

FIG. 2. As in Fig. 1, but now for $r_{s}^{2 \mathrm{D}}=4\left(L_{\max }=15.39\right)$. 
correlation energy per electron tends to zero as the quantum-well thickness $L_{\max } / \lambda$ goes to zero; for ISTLS, it tends to a realistic negative value.

In summary, we have shown that the ISTLS approach [3] correctly and remarkably describes the dimensional crossover (from 3D to 2D) of the xc energy. The quasi-2D electron gas is an important and difficult test for density functional approximations. This test is related to the onedimensional scaling, an exact constraint which is not satisfied by LSDA, GGA, or meta-GGA. The fourth-rung hyper-GGA with full exact exchange [see Eq. (1)] is found to improve considerably the behavior of local and semilocal density functionals over the whole thickness range of the quasi-2D electron gas. The fifth-rung ISTLS scheme, which uses as input all occupied and unoccupied KS orbitals and is numerically more expensive [35], is found to be remarkably accurate for the description of various quasi-2D systems (where second-order correlation can suffice [36]). Because of its accuracy in describing both the jellium surface energy [26] and the energy per particle of a quasi-2D electron gas, we believe that further work in testing and improving the ISTLS scheme could be important. Thus far, the computational cost of ISTLS has limited its applications to situations in which the density variation is effectively 1D [26]. Some chemical tests have been carried out, however, at the RPA level [37], and these calculations could well be extended within the full ISTLS scheme.

L. A.C. and J.P.P. acknowledge NSF support (Grant No. DMR05-01588), and J.M.P. acknowledges support by the Spanish MEC and the EC NANOQUANTA. L. A.C. thanks the Donostia International Physics Center (DIPC), where this work was started.

[1] A Primer in Density Functional Theory, edited by C. Fiolhais, F. Nogueira, and M. Marques, Lecture Notes in Physics (Springer, Berlin, 2003).

[2] W. Kohn and L. J. Sham, Phys. Rev. 140, A1133 (1965).

[3] J.F. Dobson, J. Wang, and T. Gould, Phys. Rev. B 66, 081108 (2002).

[4] Physical systems with strong 2D character are found [5], e.g., in the widely used semiconductor heterojunctions, in electrons bound to the surface of liquid helium, and in the copper-oxide planes of high-temperature superconductors.

[5] T. Ando, A. B. Fowler, and F. Stern, Rev. Mod. Phys. 54, 437 (1982); see also Ref. [14].

[6] J. P. Perdew and K. Schmidt, in Density Functional Theory and its Application to Materials, edited by V. Van Doren et al. (AIP, Melville, NY, 2001).

[7] J. P. Perdew and Y. Wang, Phys. Rev. B 45, 13244 (1992).

[8] J. P. Perdew, K. Burke, and M. Ernzerhof, Phys. Rev. Lett. 77, 3865 (1996); J. P. Perdew et al., Phys. Rev. Lett. 100, 136406 (2008).

[9] J. Tao et al., Phys. Rev. Lett. 91, 146401 (2003).

[10] S. Kurth, J. P. Perdew, and P. Blaha, Int. J. Quantum Chem. 75, 889 (1999); V. N. Staroverov et al., Phys. Rev. B 69, 075102 (2004).
[11] K. Rytkönen, J. Akola, and M. Manninen, Phys. Rev. B 75, 075401 (2007); M.C. Valero, P. Raybaud, and P. Sautet, Phys. Rev. B 75, 045427 (2007).

[12] S. M. Reimann and M. Manninen, Rev. Mod. Phys. 74, 1283 (2002).

[13] L. Pollack and J. P. Perdew, J. Phys. Condens. Matter 12, 1239 (2000).

[14] Y. Kim et al., Phys. Rev. B 61, 5202 (2000).

[15] P. García-González, Phys. Rev. B 62, 2321 (2000); P. García-González and R. W. Godby, Phys. Rev. Lett. 88, 056406 (2002).

[16] J. P. Perdew et al., Phys. Rev. A 76, 040501(R) (2007).

[17] J. Tao et al., Phys. Rev. A 77, 012509 (2008).

[18] E. K. U. Gross, J. F. Dobson, and M. Petersilka, in Density Functional Theory II, Topics in Current Chemistry Vol. 181, edited by R.F. Nalewajski (Springer, Berlin, 1996), p. 81 .

[19] E. K. U. Gross and W. Kohn, Phys. Rev. Lett. 55, 2850 (1985).

[20] J. Harris and A. Griffin, Phys. Rev. B 11, 3669 (1975); D. C. Langreth and J. P. Perdew, Phys. Rev. B 15, 2884 (1977); 21, 5469 (1980); 26, 2810 (1982); O. Gunnarsson and B. I. Lundqvist, Phys. Rev. B 13, 4274 (1976).

[21] In a variety of metal surfaces, the partially occupied band of Shockley surface states with energies near the Fermi level forms a quasi-2D electron gas that is immersed in a semi-infinite 3D gas of valence electrons. This system was investigated in Ref. [22] using the RPA.

[22] J. M. Pitarke et al., Phys. Rev. B 70, 205403 (2004); B. Diaconescu et al., Nature (London) 448, 57 (2007).

[23] L. A. Constantin and J.M. Pitarke, Phys. Rev. B 75, 245127 (2007), and references therein.

[24] K. S. Singwi et al., Phys. Rev. 176, 589 (1968).

[25] T. J. M. Boyd and J.J. Sanderson, Plasma Dynamics (Barnes and Noble, New York, 1969).

[26] L. A. Constantin et al., Phys. Rev. Lett. 100, 036401 (2008).

[27] The 3D parameter $r_{s}$ is the radius of a sphere containing on average one electron; $r_{s}^{2 \mathrm{D}}$ represents the radius of a circle that encloses on average one electron.

[28] M. R. Hedayati and G. Vignale, Phys. Rev. B 40, 9044 (1989).

[29] M. Seidl, J. P. Perdew, and M. Levy, Phys. Rev. A 59, 51 (1999), and references therein.

[30] D. M. Newns, Phys. Rev. B 1, 3304 (1970).

[31] H. Ou-Yang and M. Levy, Phys. Rev. A 42, 155 (1990); M. Levy and H. Ou-Yang, ibid. 42, 651 (1990).

[32] For $L>L_{\max }$, four or five cycles yield a converged solution. For $L \ll L_{\max }$, however, 10 or 11 cycles are needed in order to obtain well-converged results.

[33] M. Jonson, J. Phys. C 9, 3055 (1976).

[34] The RPA is known to yield both 2D and 3D correlation energies that are too negative. In the case of a slab, the correlation energy of both the slab and the corresponding bulk material are too negative, yielding surface energies that are very close to more refined calculations [26].

[35] The computational time for the present calculations is less than 1 min per $L$ value for LSDA and PBE, and less than $10 \mathrm{~min}$ for HGGA, but can be more than $6 \mathrm{~h}$ for ISTLS.

[36] S. Rigamonti and C. R. Proetto, Phys. Rev. B 73, 235319 (2006).

[37] F. Furche, Phys. Rev. B 64, 195120 (2001). 